\title{
How to Build Consumers' Adoption Preference for Third-Party Mobile Payment Platform
}

\author{
Weihong Zhao, Shengcheng Xie \\ School of Business, Jiangxi Normal University \\ Jiangxi, China
}

\begin{abstract}
Understanding how to obtain consumer adoption preferences is the basis for third-party mobile payment platform to gain a competitive advantage in the payment market. Based on customer perceived value theory, this study used in-depth interviews and empirical test methods to construct a research model of perceived value driving third-party mobile payment platform to obtain consumer adoption preferences. Evidence shows that process value perception (perceived ease of use, perceived risk and perceived convenience) and result value perception (perceived usefulness, perceived pleasure) effect thirdparty mobile payment platform to obtain consumer adoption preference through perceived value. The relative influence of perceived adoption preference on consumers is perceived pleasure, perceived convenience, perceived ease of use, perceived risk, and perceived usefulness. These conclusions provide theoretical and management implications for the construction of third-party mobile payment platforms and other payment platforms.
\end{abstract}

Keywords-Third-party mobile payment platform; Customer perceived value; Adoption preference

\section{INTRODUCTION}

As the main force of Internet and financial ecology, thirdparty mobile payment platform has become the mainstream platforms for individual payment and transfer. How to enhance users' stickiness and adoption preference is an important issue faced by emerging third-party mobile payment platform. The existing research mainly discusses the influence factors of consumers' adoption preference from consumers' own perspective and external stimulus perspective [1-4]. However, there are still few studies on the key factors and mechanism of adoption preference formation. In fact, the use of mobile payment platforms is not merely a question of acceptance by consumers, but also a process of value balance between gains and losses [5]. Consumers use payment platform as service experience, and the perceived value that they generate comes from the environment, processes, results, and remedies throughout the platform's use [6]. In the era of internet virtual finance, people are more concerned with the feelings and expectations of using payment platforms. Therefore, this study will explore prepositional factors of consumer perceived value from third-party mobile payment platform through the perspectives of process perceived and result perceived to reveal the mechanism model of third party mobile payment platform obtain consumers' adoption perference, and to provide management inspiration for third-party mobile payment platform and other payment platforms.

\section{THEORETICAL BACKGROUND}

Perceived value is the overall evaluation of product or service by consumer after balancing perceived benefit and cost [7]. Wolfgang divided the perceived value elements into the perception of product characteristics, service characteristics and promotion characteristics. Among them, the perception of product characteristics includes the perception of availability and ease of use of product attributes; the perception of service characteristics includes the perception of technical support, technological innovation, reliability, speed of supply and technical information; the perception brought by price reduction, red envelopes and image integration has formed the perception of promotional characteristics. These factors of perceived value are the source of competitive advantage for enterprises, but they are too fragmented to make it easier for companies to develop perceived value for consumers. This study has refined the consumer perceived value elements into perceived ease of use, perceived risk, perceived convenience, perceived usefulness and perceived pleasure. According to the theory of customer perceived value, consumers' use or re-use depends on the perceived value after balancing benefits and losses by consumers. Thus, this study believes that perceived value will drive third-party mobile payment platform to gain consumer adoption preference.

\section{RESEARCH MODEL AND RESEARCH METHODS}

\section{A. Research model.}

Based on the above background, this study proposes a research model in which perceived value drives third-party mobile payment platform to obtain consumer preferences, as shown in Fig. 1. In the entire process of consumers using thirdparty mobile payment platforms, perceived ease of use, perceived risk, and perceived convenience constitute process value perception, perceived usefulness, and perceived pleasure constitute perceived value perception. And it assumes that the perceived ease of use, perceived convenience, perceived usefulness, and perceived pleasure obtained by consumers from third-party mobile payment platform are positively correlated with perceived value, and perceived risk is negatively correlated with perceived value, and perceived value is 
positively related to consumer adoption preference of thirdparty mobile payment platform.

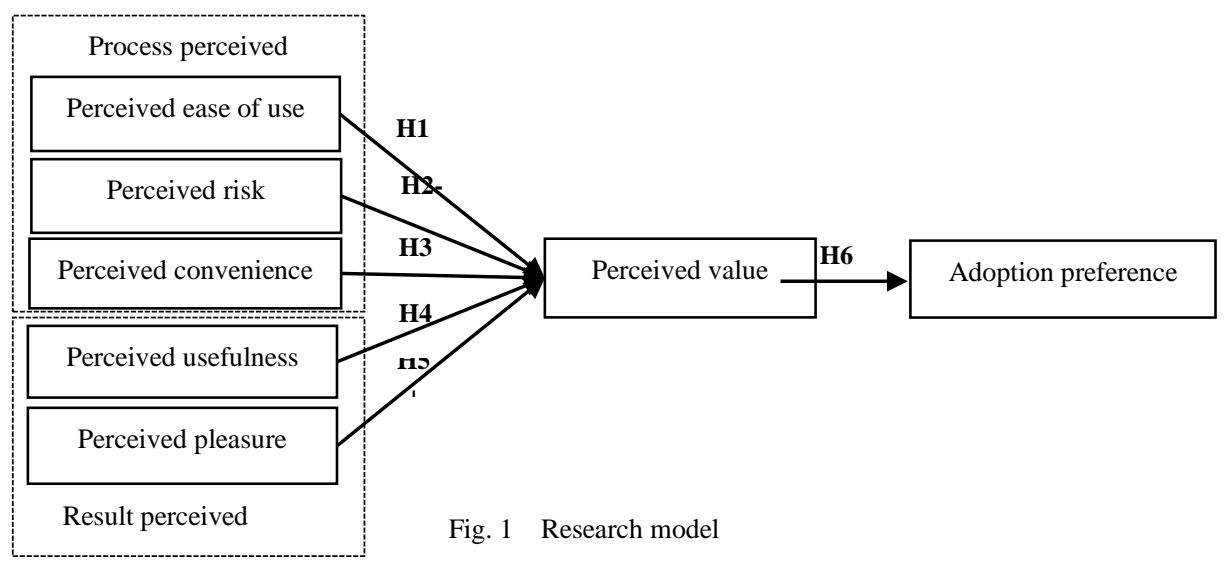

\section{B. Measurement}

In order to ensure the validity and reliability of the questionnaire, this study draws on the maturation scale of relevant foreign literature, and then combines the context of this article with the in-depth interview data to modify the measurement items. First, referring to foreign scales, four items were designed for perceived ease of use, perceived usefulness, perceived pleasure, perceived value and adoption preference, and five items were designed for perceived risk.[8 -12] Second, this study conducted a group interview with 30 randomly selected consumers who have experience with third-party mobile payment platform and other payment platforms. Finally, through the collation of interview data, the elements are coded and classified, and the applicability and rationality of the questionnaire are evaluated, the questionnaire are revised and adjusted to the final questionnaire.

\section{Data collection and sample characteristics}

This study adopts the form of questionnaires issued on the ground and online questionnaires. The content of the questionnaire is divided into two parts. One part is the use of a Likert scale to measure the driving factors that third-party mobile payment platform obtain consumer preference, and the other is the collection of personal information of survey respondents. The survey participants used five levels for each item $(1=$ completely disagree; $2=$ less agree; $3=$ not sure; $4=$ somewhat agree; $5=$ completely agree). According to statistics, total of 400 questionnaires were issued, with a total of 272 valid questionnaires. Of the respondents, $52.9 \%$ were females and $43.1 \%$ were males; $87.1 \%$ were $18-45$ years old and $8.5 \%$ were over $45 ; 72.1 \%$ were college students, and $11.8 \%$ were graduates or higher. As for monthly mobile phone charges, $54.4 \%$ were under 50 yuan, and only $4.4 \%$ were over 150 yuan; $63.2 \%$ of respondents use it more than 8 times per week, and $22.1 \%$ use it between 3-8 times per week.;42.3\% came from Jiangxi, $22.4 \%$ came from Guangdong, and $21.3 \%$ came from Hubei.

\section{RESULTS}

\section{A. Measurement evaluation.}

This study used AMOS 7.0 to perform confirmatory factor analysis on 7 multi-item models consisting of 28 items. As a result, one item was deleted for each perceived ease of use, perceived convenience, perceived usefulness, and perceived value. The overall measurement model fit was significantly optimized. The results of the analysis are shown in Table 1. It can be seen from the fitness index that the overall degree of the model is better; the Cronbach's a values are all greater than 0.7 , and the internal consistency reliability of each latent variable is relatively high; secondly, the Normalized load factors of all latent variables are greater than 0.5 , the average extraction variance (AVE) values are all greater than 0.5, and the combined reliability $(\mathrm{CR})$ are all greater than 0.7 , indicating that the latent variables have high concentration validity; at the same time, as shown in Table 2, the average variance (numbers on the diagonal) of each latent variable is greater than the square of the correlation coefficient between the latent variables (numbers on the non-diagonal lines), indicating that the discriminant validity between the latent variables is high and the latent variables are irrelevant with each other. 
TABLE I. MEASUREMENT MODEL RESULTS

\begin{tabular}{|c|c|c|c|c|c|c|c|}
\hline & 1 & 2 & 3 & 4 & 5 & 6 & 7 \\
\hline 1. Perceived ease of use & 0.63 & & & & & & \\
\hline 2.perceived risk & 0.45 & 0.73 & & & & & \\
\hline 3.perceived convenience & 0.01 & 0.06 & 0.71 & & & & \\
\hline 4.perceived usefulness & 0.32 & 0.44 & 0.10 & 0.71 & & & \\
\hline 5.perceived pleasure & 0.27 & 0.59 & 0.45 & 0.05 & 0.72 & & \\
\hline 6.perceived value & 0.00 & 0.01 & 0.48 & 0.10 & 0.54 & 0.74 & \\
\hline 7.adoption preference & 0.02 & 0.27 & 0.58 & 0.31 & 0.55 & 0.06 & 0.69 \\
\hline Cronbach's $\alpha$ & 0.74 & 0.83 & 0.88 & 0.84 & 0.75 & 0.80 & 0.89 \\
\hline C.R & 0.84 & 0.91 & 0.88 & 0.88 & 0.91 & 0.90 & 0.90 \\
\hline AVE & 0.63 & 0.73 & 0.71 & 0.71 & 0.72 & 0.74 & 0.69 \\
\hline
\end{tabular}

${ }^{\mathrm{a} .} \chi(\mathrm{P}) / \mathrm{df}=3.6 ; \mathrm{CFI}=0.90 ; \mathrm{NFI}=0.81 ; \mathrm{GFI}=0.95 ; \mathrm{RMR}=0.04 ; \mathrm{AGFI}=0.91$

\section{B. Hypothesis testing.}

The structural equations of the model were analyzed by AMOS7.0. The fitting indicators showed that the structural model had a good degree of fit. The assumptions of this study were also supported. The results are shown in Table 2. In process perceived and result perceived of consumers using third-party mobile payment platform, perceived risk has a negative impact on perceived value; perceived ease of use, perceived convenience, perceived usefulness and perceived pleasure have a certain positive impact to perceived value. But perceived usefulness does not show a significant positive effect on perceived value. This may be due to the fact that third-party mobile payment platform and other payment platforms have similar benefits to consumers' life or work, so consumers are less sensitive to the perceived usefulness of third-party mobile payment platform. The perceived value obtained by consumers from third-party mobile payment platform is positively related to adoption preferences.

TABLE II. HYPOTHESIS TEST RESULTS

\begin{tabular}{|c|c|c|c|}
\hline Hypothesis & Estimate & C.R. & Validation results \\
\hline H1 & 0.517 & 3.146 & Support*** \\
\hline H2 & -0.354 & -2.582 & Support*** \\
\hline H3 & 0.723 & 5.995 & Support*** \\
\hline H4 & 0.138 & 1.925 & Partial support** \\
\hline H6 & 0.916 & 8.210 & support*** \\
\hline
\end{tabular}

b. $\mathrm{CFI}=0.93 ; \mathrm{NFI}=0.81 ; \mathrm{GFI}=0.96 ; \mathrm{RMR}=0.04 ; \mathrm{AGFI}=0.94$

c. Note:*indicates $\mathrm{P}<0.1 ; * *$ indicates $\mathrm{P}<0.05$; ***;indicates $\mathrm{P}<0.001$

\section{CONCLUSIONS}

This study established a model that perceived value drives third-party mobile payment platform to gain consumers' adoption preferences, and has achieved interesting conclusions. First, the process by which third-party mobile payment platform gain consumer adoption preferences is the process of perceived value formation. Third-party mobile payment platform need to focus on increasing consumers' perceived sensitivity to the value of third-party mobile payment platform in order to obtain consumer adoption preference. Second, consumers' perceived ease of use, perceived risk, perceived convenience, perceived usefulness, and perceived pleasure derived from third-party mobile payment platform create perceived value. Among them, perceived pleasure has the strongest influence on consumer adoption preference, followed by perceived convenience, perceived ease of use, perceived risk, and perceived usefulness. This shows that with the development of technology, whether the basic functions of third-party mobile payment platform can meet the daily needs of consumers and the risks of the platform are not the primary concerns of consumers. Instead, consumers pay special attention to the pleasure of red envelopes and rebates when using third-party mobile platform.
This study extends the applicability of customer perceived value theory to perceived value drive consumer adoption preference, and helps explain the inherent mechanism of third party mobile payment platforms obtain consumer adoption preference. First, this study focuses on consumer adoption preference and perceived value analysis, examines the perceived value elements, and explains the relationship between perceived value and consumer behavior based on the customer perceived value theory. Secondly, this study draws on the research of scholars in the field of service marketing, and places perceived value as a service experience in process perception and result perception in search of pre-influencing factors, thereby enriching the existing research path. Finally, this study focuses on the value-balancing aspect of adoption preference formation process and establishes a research model that reveals the key elements and mechanisms of perceived value driving third-party mobile payment platforms to obtain consumer adoption preference, further deepening the internal mechanism of third party Mobile payment platforms gain consumer adoption preference.

In order to enhance users' stickiness and gain consumer preference, third-party mobile payment platform and other developers can start from the following points. First, Perceived value is not only a measure of the balance between gains and losses brought about by consumers and external stimuli, but also a predictor of consumer preferences. Designers of third- 
party mobile payment platform should maximize the use of their existing technologies to enhance consumers' perceived value of third-party mobile payment platform by enhancing the subsidiary functions of platform, simplifying the use of processes, and strengthening payment security. At the same time, due to the different needs of consumers, platform developers should develop different versions (such as the simplified version and the normal version) of the payment platform or customize different payment platform affiliate functions for different customers based on different consumer groups to meet consumers' needs. Second, payment service providers must not only pay attention to the perceived value brought by the platform technology level, but also emphasize the perceived value of platform marketing strategies, and rationally allocate various perceived value elements to maximize perceived value of the platform for consumers. And further enhances the consumer's use of third-party mobile payment platform.

Although this study has achieved some important research conclusions, this study still has the following deficiencies. First there are many factors that influence the third party mobile payment platform to obtain consumer preference. This study only validates the role of some elements from the perspective of perceived value, and other elements are not included in the model. Second, the entire process of using third-party mobile payment platforms by consumers includes the entire experience of environment, processes, results and remedies. This study only explores the pre-influencing factors of perceived value from the perspective of process and result. Third, the survey sample has limitations. Due to time and energy constraints, indepth interviews and questionnaire surveys are mostly for university students. Defects in sampling may have an impact on the conclusions. However, undergraduates are the main group of people using third-party mobile payment platforms, which to some extent make up for the lack of sampling.

\section{ACKNOWLEDGMENT}

This research was supported by the college student project of Jiangxi Normal University.

\section{REFERENCES}

[1] Kurt B. Waldman, John M. Kerr. Does safety information influence consumers' preferences for controversial food products?[J]. Food Quality and Preference,2018,64:56-65.

[2] Shilpa S. Samant, Matthew J. Chapko, Han-Seok Seo. Predicting consumer liking and preference based on emotional responses and sensory perception: a study with basic taste solutions[J] Food Research International,2017,100(1):325-334

[3] Chia-Li Lin, Chun-Wen Chen, Gwo-Hshiung Tzeng. Planning the development strategy for the mobile communication package based on consumers' choice preferences [J]. Expert Systems with Applications, 2010,37(7):4749-4760.

[4] Michelle D. Steward, James A. Narus, Michelle L. Roehm. An exploratory study of business-to-business online customer reviews: external online professional communities and internal vendor scorecards $[\mathrm{J}]$. Journal of the Academy of Marketing Science,2018,46(2):173-189.

[5] Tomi Dahlberg, Niina Mallat, Jan Ondrus. Past, present and future of mobile payments research:a literature review[J].Electronic Commerce Research and Applications,2018,7:165-181.

[6] Zhao Weihong, Xiong Xiaoming. Measurement and management of online retail service quality based on chinese situation [J]. Management review,2015, 27(12). (In Chinese)

[7] Wolfgang Ulaga. Customer value in business markets[J].Industrial Marketing Management,2001,30(4):315.

[8] Zhao Weihong. The construction of customer loyalty in online retailfocusing on the relationship between value perception, satisfaction experience and trust, and relative influence[J]. Journal of Business Economics,2007,5, 1000-2154. (In Chinese)

[9] Vess L. Johnson, Angelina Kiser, Ronad Washington. Limitations to the rapid adoption of M-payment services: Understanding the impact of privacy risk on M-Payment services[J]. Computers in Human Behavior,2018,79:111-122.

[10] Chatterjee P, Rose R L. Do payment mechanisms change the way consumers perceive products?[J]. Journal of Consumer Research,2012,38(6):1129-1139.

[11] Zhao Weihong. Customer value in online retail and its influence on store loyalty [J]. Economic Management Journal, 2017,5:1002-5766. (In Chinese) 